\title{
Exoplanet Surveys at Universidad de Chile
}

\author{
Astronomy Department, Universidad de Chile, \\ Casilla 36-D, Santiago, Chile \\ contact email: pato@oan.cl
}

Patricio Rojo, James Jenkins, Sergio Hoyer and Matías Jones

\begin{abstract}
We present and highlight the first results of the three main exoplanet surveys we are currently conducting at Universidad de Chile: CHEPS, Red Giant Exoplanets (radial velocity), and TraMoS (transit lightcurves). We have several interesting candidates at the CalanHertfordshire Extrasolar Planet Search (CHEPS) project, which is aimed at searching for the currently missing southern bright transiting planets at a few $\mathrm{m} / \mathrm{s}$ radial velocity precision. Using the same technique, we are also characterizing the planetary population in a constrained sample of Red Giant stars. The Transit Monitoring from the South (TraMoS) project is aimed both at improving transit parameters and at detecting any kind of lightcurve variability from several known southern exoplanet systems.
\end{abstract}

Keywords. planets: general, surveys, techniques: radial velocity, techniches: photometric

\section{Introduction}

Sunrise of a gigantic red sun or sunsets of two different suns are no longer in the sole realm of fiction. In the almost 1000 extrasolar planets discovered to this date, the variety of orbital configurations and planetary characteristics has been closer to what science fiction writers have imagined than what theoretical models of planetary system formation expected.

Thanks to the maturity of techniques and the increasing time span of surveys, new planets are being discovered every month. Nevertheless, the real challenge is to be able to characterize extrasolar planets. Until little over 15 years ago, our planetary knowledge was limited to our own Earth, the 8 other planets orbiting our sun, all the satellites, and the myriad of small bodies like asteroids and comets. In those times, the theories of planetary formation and evolution were very consistent with observations. However, ever since the first discoveries of extrasolar planets, those theories have been challenged, improved, and/or replaced. For instance, the now classical debate between the core accretion vs gravitational instability formation scenarios is far from settled (Boss 2011; Boley 2009, and references therein). Even the $10+$ years uncontested planetary migration theory that was quickly summoned as an explanation for Hot-Jupiters (Lin et al. 1996), is now challenged by cumulative observations of the Rossiter-McLaughlin effect seem to indicate that, instead, most hot Jupiters have dynamical/tidal origins (Triaud et al. 2010).

Since most of the known extrasolar planets have been discovered by the radial velocity method, only bulk properties are known for them. More precisely, the planetary information is limited to the orbital period (and the semi-major axis of the orbit as a direct consequence of Kepler's laws) and an upper limit to the planetary mass: $M \sin i$, where $i$ is the unknown orbital inclination. However, the growing population of transiting planets, have not only provided a precise determination of exoplanet's masses, but have provided a fast track to learn about planetary and atmospheric properties like their radius, thermal emission, albedo, atmospheric components, and have thus been studied extensively (e.g. Charbonneau et al. 2002; Tinetti et al. 2007; Swain et al. 2008; Bean et al. 2010; 
Rojo 2006). From these studies, it has become clear that planets are not just small stars, since not even their bulk properties are solely determined by their masses. In fact, many observations are yet to be explained by a satisfactory theoretical framework, among them are the inflated hot-jupiters or the non-ubiquitous inversion layers (e.g. Fortney et al. 2008; Laughlin et al. 2011; Madhusudhan et al. 2011).

Direct measurement of atmospheric abundances could provide some of the best constraints on models; however, it is challenging to detect the weak planetary signal in the Poisson noise from the strong stellar flux. Photometric, or broad band, observations are sensitive to small planet-to-star flux ratios. However, photometric observations are not nearly as good as spectroscopic observations to constrain abundances. The importance of studying transiting systems is further evidenced by the more than 900 publications that have been produced on the 2 brightest transiting planets, HD 209458b and HD 189733b, first detected by RV surveys (Charbonneau et al. 1999; Bouchy et al. 2005).

Precise atmospheric characterization thus requires bright transiting stars but, inexplicably, all the brightest transiting stars have northern declinations. Only the 12th brightest transiting system is in the southern hemisphere, more than 3 magnitudes fainter than the brightest system.

\section{Surveys}

Making use of the privileged access to state-of-the-art astronomical facilities in the Atacama dessert, the extrasolar planet group at Universidad de Chile is undergoing long-term surveys to help solve some of the above questions. They are possible under the collaboration with researchers from different institutions.

\subsection{CHEPS}

The Calan-Hertfordshire Extrasolar Planet Survey (CHEPS, PIs: J.S. Jenkins, P. Rojo, H.R.A. Jones) has been running from 2008 using the state-of-the-art planethunters HARPS and Coralie spectrographs. For the latter we had to develop our own pipeline which currently performs extremely precisely with precision well below $10 \mathrm{~m} \mathrm{~s}^{-1}$.

We aim to search for more benchmark transit planets in the southern hemisphere, focusing on the as yet untapped radial-velocity phase extremely short-period exoplanets,

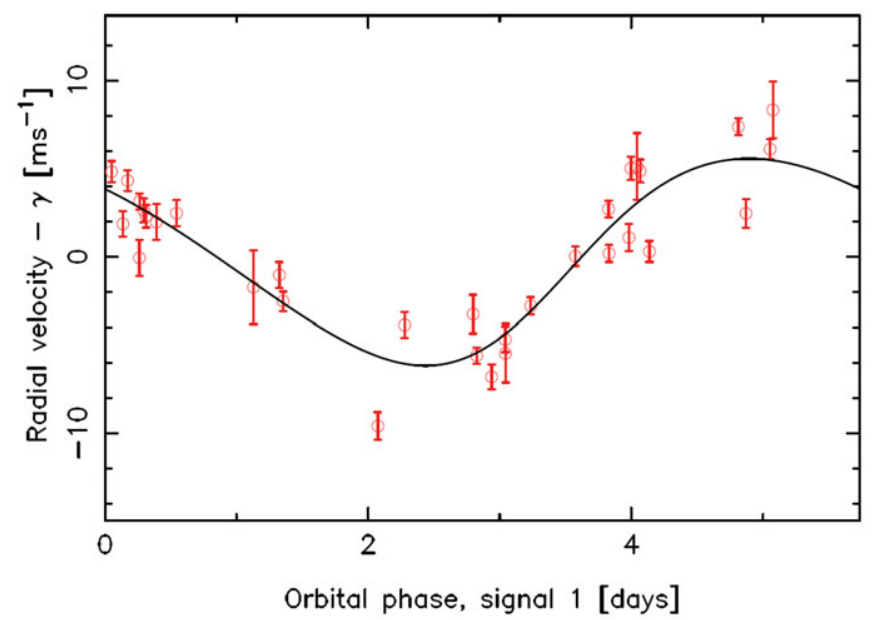

Figure 1. Phase-folded best Keplerian orbital solution for the Hot Uranus orbiting HD77338 (Jenkins et al.2013). A transit signal was searched unsuccessfully. 
with the aim of discovering more benchmark planets where follow-up can be performed to probe the physics of exoplanetary atmospheres. Adding even one bright transit planet to the small list currently known will give rise to a significant advance in our knowledge of exoplanetary atmospheres. Note that surveys like CoRoT and Kepler have detected many more transiting planets but follow-up work will be difficult due to the magnitude distribution of their target fields, meaning that in the near future radial-velocity programs will still provide the best targets for follow-up to probe exoplanetary atmospheres.

We have already published the catalog with our 850 target stars, including detailed activity and kinematics analysis (Jenkins et al. 2011); the detection of a very eccentric brown dwarf in the dessert (Jenkins et al. 2009); and the detection of a Hot-Neptune together with a possible new metallicity trend for low-mass planets (Fig. 1, Jenkins et al. 2013). Perhaps even most interesting is the presence of a RV signal above the instrumental noise in $\sim 40 \%$ of the sample.

\subsection{Red Giants}

During the last few years we have also been conducting a radial velocity survey aimed at studying the population of close-in planets orbiting giant stars (PIs: M. Jones, J. Jenkins, P. Rojo). The overarching aim is to establish whether the missing close-in are primordial or evolutional.

So far, we have collected multi-epoch spectra for a sample of 164 bright GK giant stars (Jones et al. 2011, Fig. 2). We used the spectrographs FECH+Chiron (at the CTIO $1.5 \mathrm{~m}$ telescope) and FEROS at the La Silla $2.2 \mathrm{~m}$ telescope. The former using and Iodine cell for calibration while the latter ThAr fiber. Several exciting planetary candidates are currently being investigated.

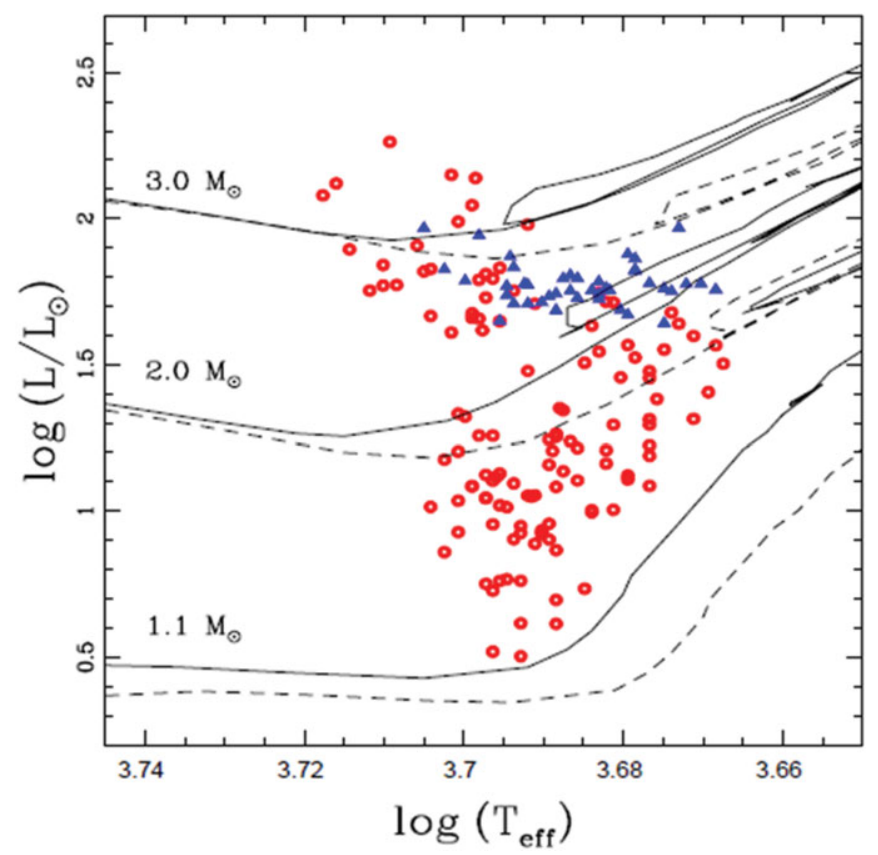

Figure 2. HR diagram including all of our red giant survey targets. The red open circles correspond to RGB stars, while the blue filled triangles to HB stars. Different evolutionary tracks (Salasnich et al. 2000) are overplotted for stars with $1.1 \mathrm{M}_{\odot}, 2.0 \mathrm{M}_{\odot}$ and $3.0 \mathrm{M}_{\odot}$ (line pairs from bottom to top). The solid lines correspond to models with $[\mathrm{Fe} / \mathrm{H}]=0.0$ and the dashed lines to $[\mathrm{Fe} / \mathrm{H}]=0.32$ 


\section{3. $\operatorname{Tr} a M o S$}

The Transit Monitoring in the South project (PIs: P. Rojo, S. Hoyer, M. López-Morales) has been running since 2008. Its main objective is the search for the variability of transiting parameters. In particular, the monitoring of variability in the time of the transit's center (TTVs) is now a proved technique to constrain the masses of unseen planetary companions, albeit no firm such detection has yet been announced for ground-based discovered system. This is a long-term project that is also expected to present shortterm results, which would be quickly processed given our working pipelines. In addition, the combined lightcurve will provide improved parameters of (among others) planetary radius and inclination for all of our systems.

The sample comprises observable southern transiting systems $(\delta<+10)$, most of them brighter than $V \sim 13$ magnitude. Depending on magnitude, the monitoring has been done

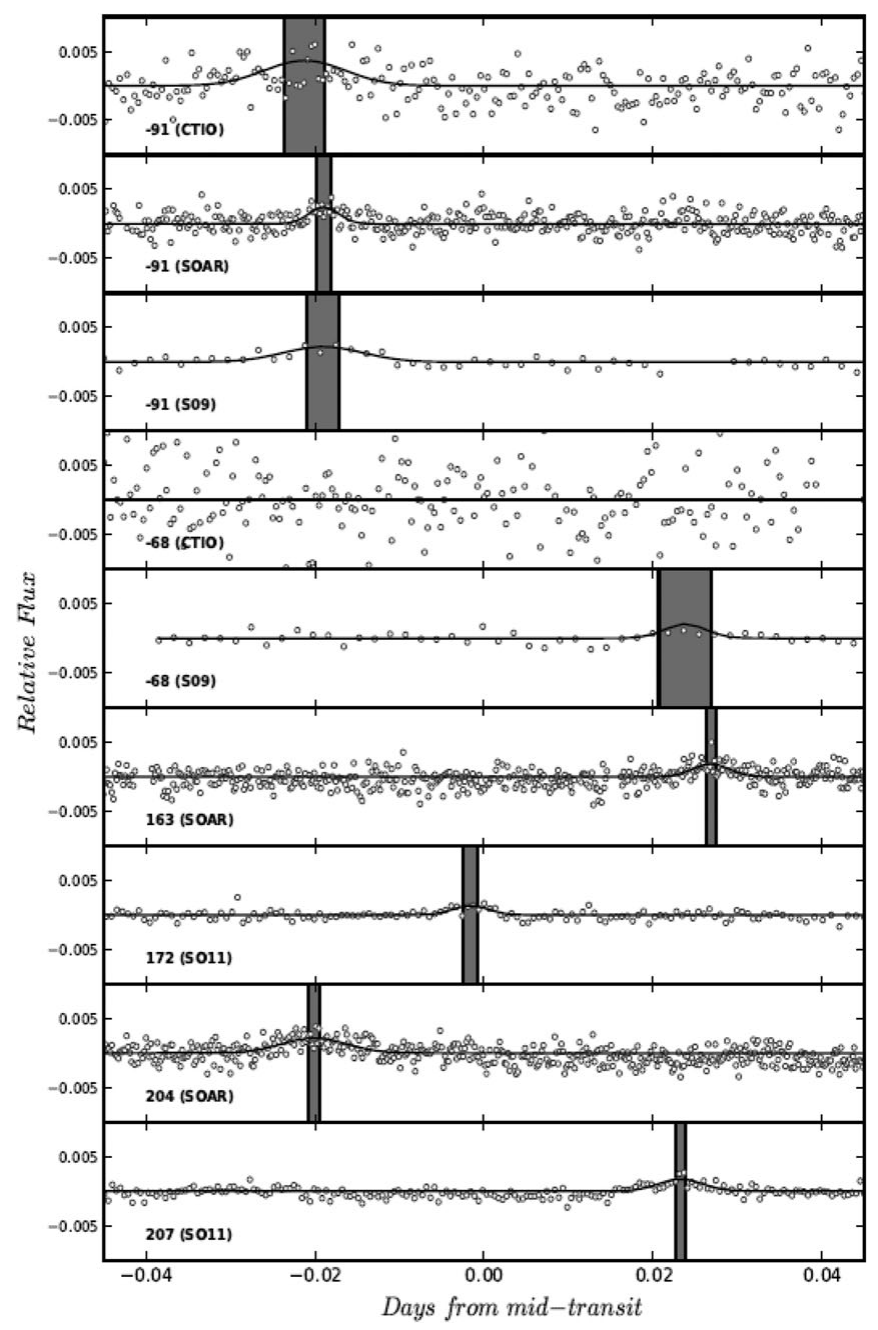

Figure 3. Residual light curves with evidences of star-spot occultations by WASP-4b. The epoch and telescope initial of the light curve are shown in the bottom left. The solid lines represent the fitted gaussian model and the gray regions are proportional to the uncertainties in the timing of the occultations. We confirm Sanchis-Ojeda et al. (2011)'s detection of the occultation with our -91 epochs data but in our -68 transit we found no evidence of occultation due the low SNR of the data. 
with 0.6-, 1-, 4-, and even 8-meter telescopes, respectively providing the required superb accuracy. Currently, almost 80 transits have been observed on over 20 known planets. Additionally, we search for transits to CHEPS candidates.

We have already firmly refuted previous claims of TTV detection on OGLE-TR-111 and WASP-5 (Hoyer et al. 2011, 2012), and are proposing better constraints on stellar spots for WASP-4 (Fig 3, Hoyer et al 2013)

\section{Conclusions}

The exoplanet surveys at Universidad de Chile have been very successful at securing observing time at state-of-the-art telescopes and are reaching maturity with highly complementary aims. We already have several publications, but robust pipelines and preliminary analysis presume many more upcoming interesting results.

\section{References}

Bean, J. L., Miller-Ricci Kempton, E., \& Homeier, D. A ground-based transmission spectrum of the super-Earth exoplanet GJ 1214b. Nature, 468: 669-672, December 2010

Boley, A. C. The Two Modes of Gas Giant Planet Formation. ApJL, 695: L53-L57, April 2009

Boss, A. P. Formation of Giant Planets by Disk Instability on Wide Orbits Around Protostars with Varied Masses. ApJ, 731: 74, April 2011

Bouchy, et al. A\&A, 444: L15, December 2005

Charbonneau, D., et al. IAU Circular 7513, 1999

Charbonneau, D., Brown, T. M., Noyes, R. W., \& Gilliland, R. L. Detection of an Extrasolar Planet Atmosphere. ApJ, 568: 377-384, March 2002.

Fortney, J. J., Lodders, K., Marley, M. S., \& Freedman, R. S. A Unified Theory for the Atmospheres of the Hot and Very Hot Jupiters: Two Classes of Irradiated Atmospheres. ApJ, 678: 1419-1435, May 2008

Hoyer, S., Rojo, P., López-Morales, M., Díaz, R. F., Chambers, J., \& Minniti, D. Five New Transit Epochs of the Exoplanet OGLE-TR-111b. ApJ, 733: 53, May 2011

Hoyer, S., Rojo, P., \& López-Morales, M. Transit Monitoring in the South (TraMoS) Project: Discarding Transit Timing Variations in WASP-5b. ApJ, 748: 22, March 2012

Hoyer, S., et al. MNRAS, 434: 46, September 2013

Jenkins, J. S., Jones, H. R. A., K. Goździewski, Migaszewski, C., Barnes, J. R., Jones, M. I., Rojo, P., Pinfield, D. J., Day-Jones, A. C., \& Hoyer, S. First results from the CalanHertfordshire Extrasolar Planet Search: exoplanets and the discovery of an eccentric brown dwarf in the desert. MNRAS, 398: 911-917, September 2009

Jenkins, J. S., Murgas, F., Rojo, P., Jones, H. R. A., Day-Jones, A. C., Jones, M. I., Clarke, J. R. A., Ruiz, M. T., \& Pinfield, D. J. Chromospheric activities and kinematics for solar type dwarfs and subgiants: analysis of the activity distribution and the AVR. $A \& A, 531$ : A8, July 2011

Jenkins, J. S., et al. ApJ, 766: article id. 67, April 2013

Jones, M. I., Jenkins, J. S., Rojo, P., \& Melo, C. H. F. Study of the impact of the post-MS evolution of the host star on the orbits of close-in planets. I. Sample definition and physical properties. A\&A, 536: A71, December 2011

Laughlin, G., Crismani, M., \& Adams, F. C. On the Anomalous Radii of the Transiting Extrasolar Planets. ApJL, 729: L7, March 2011

Lin, D. N. C., Bodenheimer, P., \& Richardson, D. C. Orbital migration of the planetary companion of 51 Pegasi to its present location. Nature, 380: 606-607, April 1996

Madhusudhan, N., Harrington, J., Stevenson, K. B., Nymeyer, S., Campo, C. J., Wheatley, P. J., Deming, D., Blecic, J., Hardy, R. A., Lust, N. B., Anderson, D. R., Collier-Cameron, A., Britt, C. B. T., Bowman, W. C., Hebb, L., Hellier, C., Maxted, P. F. L., Pollacco, D., \& West, R. G. A high C/O ratio and weak thermal inversion in the atmosphere of exoplanet WASP-12b. Nature, 469: 64-67, January 2011 
Rojo, P. M. Transit spectroscopy of the extrasolar planet HD 209458B: The search for water. $\mathrm{PhD}$ thesis, Cornell University, 2006.

Sanchis-Ojeda, R., Winn, J. N., Holman, M. J., Carter, J. A., Osip, D. J., \& Fuentes, C. I. Starspots and Spin-orbit Alignment in the WASP-4 Exoplanetary System. ApJ, 733: 127, June 2011

Swain, M. R., Vasisht, G., \& Tinetti, G. The presence of methane in the atmosphere of an extrasolar planet. Nature, 452: 329-331, March 2008

Tinetti, G., Vidal-Madjar, A., Liang, M.-C., Beaulieu, J.-P., Yung, Y., Carey, S., Barber, R. J., Tennyson, J., Ribas, I., Allard, N., Ballester, G. E., Sing, D. K., \& Selsis, F. Water vapour in the atmosphere of a transiting extrasolar planet. Nature, 448: 169-171, July 2007

Triaud, A. H. M. J., Collier Cameron, A., Queloz, D., Anderson, D. R., Gillon, M., Hebb, L., Hellier, C., Loeillet, B., Maxted, P. F. L., Mayor, M., Pepe, F., Pollacco, D., Ségransan, D., Smalley, B., Udry, S., West, R. G., \& Wheatley, P. J. Spin-orbit angle measurements for six southern transiting planets. New insights into the dynamical origins of hot Jupiters. $A \mathscr{G} A$, 524: A25, December 2010 